\title{
$32 \times 32$ silicon photonic MEMS switch with gap-adjustable directional couplers fabricated in commercial CMOS foundry
}

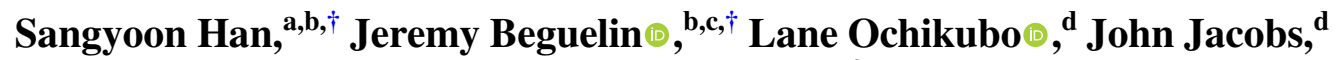 \\ Tae Joon Seok $\odot,{ }^{\text {b,e }}$ Kyoungsik Yu $\odot,{ }^{\text {b,f }}$ Niels Quack $\odot,{ }^{\text {b,g }}$ \\ Chang-Kyu Kim ${ }^{\text {b,h }}{ }^{\text {Richard S. Muller, }}{ }^{\text {b }}$ and Ming C. Wu $\odot^{\text {b,* }}$ \\ ${ }^{a}$ Daegu Gyeongbuk Institute of Science and Technology, Department of Robotics Engineering, \\ Daegu, Republic of Korea \\ ${ }^{b}$ University of California, Berkeley, Department of Electrical Engineering and \\ Computer Sciences, Berkeley, California, United States \\ ${ }^{\mathrm{c}}$ SUSS MicroOptics SA, Hauterive, Switzerland \\ ${ }^{\mathrm{d}}$ TSI Semiconductors, Roseville, California, United States \\ ${ }^{\mathrm{e}}$ Gwangju Institute of Science and Technology, School of Electrical Engineering and \\ Computer Science, Gwangju, Republic of Korea \\ ${ }^{\mathrm{f}}$ Korea Advanced Institute of Science and Technology, School of Electrical Engineering, \\ Daejeon, Republic of Korea \\ ${ }^{g}$ Institute of Microengineering, École Polytechnique Fédérale de Lausanne, Lausanne, \\ Switzerland \\ ${ }^{\text {h}}$ Korea Polytechnic University, Department of Nano and Semiconductor Engineering, \\ Siheung, Republic of Korea
}

\begin{abstract}
We report on a $32 \times 32$ silicon photonic micro-electro-mechanical-system (MEMS) switch with gap-adjustable directional couplers. The switch is fabricated on 200-mm silicon-oninsulator wafers in a commercial complementary metal-oxide-semiconductor (CMOS) foundry. The fabricated device has a maximum on-chip loss of $7.7 \mathrm{~dB}$ and an extinction ratio of $50.8 \mathrm{~dB}$. The switching voltage is $9.45 \mathrm{~V}$ and the $20-\mathrm{dB}$ bandwidth is $28.7 \mathrm{~nm}$. Our work shows a promising path for mass production of silicon photonic MEMS switches in commercial CMOS foundries. (C) The Authors. Published by SPIE under a Creative Commons Attribution 4.0 Unported License. Distribution or reproduction of this work in whole or in part requires full attribution of the original publication, including its DOI. [DOI: 10.1117/1.JOM.1.2.024003]
\end{abstract}

Keywords: silicon photonics; micro-electro-mechanical-system; switch; foundry; directional coupler.

Paper 20021 received Dec. 15, 2020; accepted for publication Mar. 5, 2021; published online Mar. 24, 2021.

\section{Introduction}

Artificial intelligence and machine learning workloads are changing all aspects of the compute infrastructure-from data centers and cloud hyperscalers to high-performance computers (HPC). ${ }^{1}$ Data centers are being enhanced with specialized HPC-like hardware to handle machine learning workloads. Hyperscale cloud infrastructure is enhanced with rack-scale and distributed solutions. Optical switching solutions have been proposed to improve the utilization of the computer resources as well as reducing latency and energy consumption. ${ }^{2-6}$ Silicon photonic switches are particularly attractive for such applications, and several research groups have developed silicon photonics switches based on cascaded Mach-Zehnder interferometers. ${ }^{7-14}$ However, their optical losses increase rapidly with port count, limiting the scalability of these switches.

Our group has previously demonstrated micro-electro-mechanical-system (MEMS)-based silicon photonic switches with moving waveguides. The switches are highly scalable and have

\footnotetext{
*Address all correspondence to Ming C. Wu,wu@eecs.berkeley.edu

${ }^{\dagger}$ These authors contributed equally to this work.
} 
several salient features. The $240 \times 240$ switch is the largest monolithic switch ever reported. ${ }^{15}$ They also have fast switching time $(<1 \mu \mathrm{s})$ and broadband operation $(>120 \mathrm{~nm})$. Polarization insensitivity has been addressed using multi-layer bus waveguides. ${ }^{16}$ However, these switches were made with custom fabrication process that are not commonly accessible. On the other hand, simpler single-layer MEMS devices can be made in standard silicon photonics process. ${ }^{17} 8 \times 8$ crossbar switches ${ }^{18}$ and $4 \times 20$ multicast switches ${ }^{19}$ have been reported, though they are not fabricated in foundries. Though the optical loss is not as low as those of more sophisticated multi-layer switches, this simple process is attractive for medium-sized switch and potential integration with other well-established silicon photonic elements including germanium photodetectors for in situ monitoring. In addition, the inclusion of silicon photonic MEMS process in foundry could open up a promising route to build programmable photonics circuits. ${ }^{20}$ In this paper, we report on a $32 \times 32$ silicon photonic MEMS switch fabricated on 200-mm wafers at a commercial complementary metal-oxide-semiconductor (CMOS) foundry (TSI Semiconductors). Switching is accomplished by directional couplers with continuously tunable gaps. The switch exhibits high uniformity, and the power consumption is several orders of magnitude lower than thermo-optic switches.

\section{Switch Design}

Figure 1 shows the architecture of the switch. It consists of a crossbar network with 32 input and 32 output waveguides. The switch is designed for a transverse electric (TE) mode operation. The waveguide crossings have been reported in Refs. 21 and 22. Grating couplers are used for input and output for ease of testing. The grating coupler is also designed for TE polarization, has a 640-nm pitch with a 50\% duty cycle, and is defined with a 70-nm-deep etch. The bandwidth of the grating coupler is $30 \mathrm{~nm}$. Each switch cell has two directional couplers that are tethered to a common comb-drive actuator moving in the diagonal direction. Similar comb drives were used in Ref. 23. The length, thickness, and width of the coupler waveguides are $20 \mu \mathrm{m}, 220 \mathrm{~nm}$, and $450 \mathrm{~nm}$, respectively. The coupling gap can be continuously varied from $550 \mathrm{~nm}$ to nearly physical contact $(\sim 0 \mathrm{~nm})$ by the comb-drive actuator. To achieve a high-density switch, the footprint of the comb drive should be minimized. At the same time, we need as many comb finger pairs as possible to reduce the switching voltage. We accomplished this using deep submicron comb fingers $(300 \mathrm{~nm})$ and spacing $(400 \mathrm{~nm})$, which yield 44 pairs of comb fingers. This gives us a switching voltage of $9.45 \mathrm{~V}$. The actuator has a footprint of $88 \mu \mathrm{m} \times 88 \mu \mathrm{m}$, well within our waveguide pitch of $166 \mu \mathrm{m}$.

Figure 2(a) shows simulated optical field $\left(E_{x}\right)$ along the directional coupler with $550 \mathrm{~nm}$ gap. In this state, the coupling between the waveguides is weak, and all light from input port goes to

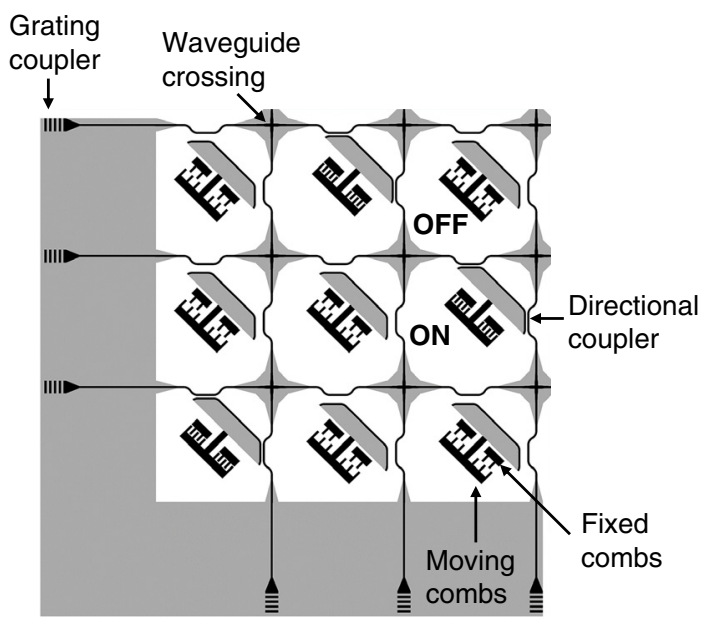

Fig. 1 The architecture of the silicon photonic MEMS switch with gap-adjustable directional couplers. Light is coupled to the chip using the grating couplers. There are two pairs of the directional couplers and one comb-drive actuator per unit cell. The light paths on the chip are controlled by changing the gap spacing of each directional coupler. 


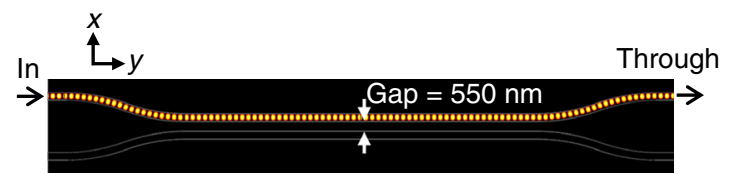

(a)

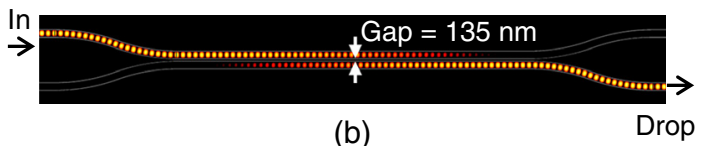

(b)

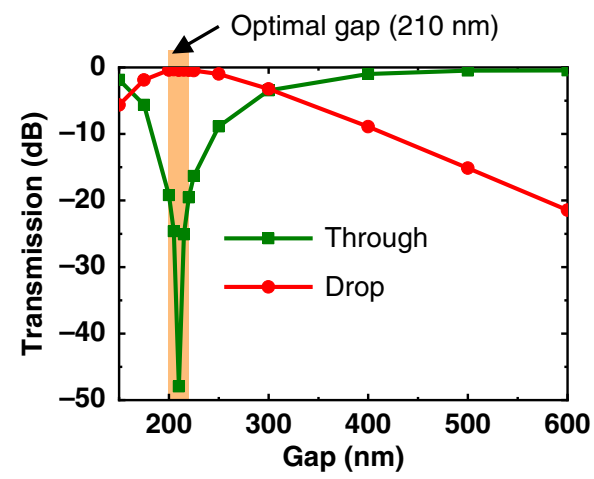

(d)

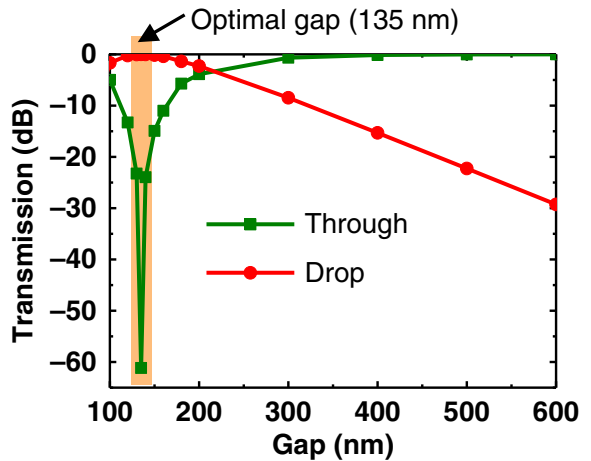

(c)

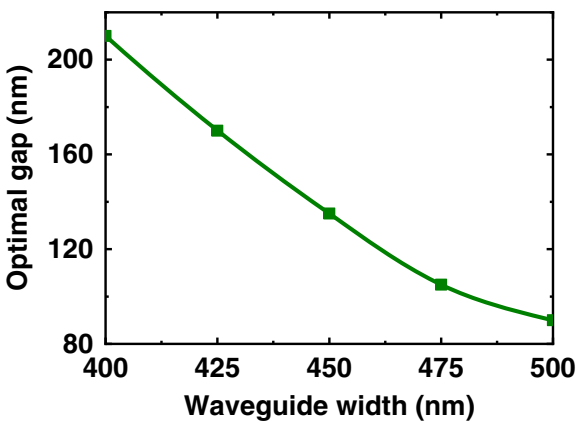

(e)

Fig. 2 Simulated optical performance of the gap-adjustable directional coupler. Unless otherwise stated, the width of the waveguide used for the simulations is $450 \mathrm{~nm}$. (a) Optical field $\left(E_{x}\right)$ profile along the directional coupler with 550-nm gap. All light from the input port goes to the through port. (b) Similar profile when the gap spacing of the directional coupler reduces to $135 \mathrm{~nm}$. Light is directed to drop port. (c) Transmission characteristics of the coupler versus gap size. (d) Transmission characteristics of the coupler when the width of the waveguide is changed to $400 \mathrm{~nm}$. (e) Optimal gap versus the waveguide width of directional couplers.

through port. Since the coupling coefficient depends exponentially on the gap spacing, the coupling increase quickly with reducing gap. At 135-nm gap spacing [Fig. 2(b)], nearly $100 \%$ of the light goes to drop port.

Figure 2(c) shows the simulated transmission of the directional coupler with various gap sizes. The simulation shows that an extinction ratio (for drop port) of $25.8 \mathrm{~dB}$ can be achieved by changing the gap size from 550 to $135 \mathrm{~nm}$. Since there are two directional couplers per switch unit cell, the extinction ratio for switching is $51.6 \mathrm{~dB}$. The switching devices using directional couplers $^{7-13}$ often suffer from optical power leakage or loss due to the dimensional shift of the directional couplers during fabrication step. However, the gap-adjustable directional coupler allows flexible tuning of the gap and thus allows compensating the dimensional shift. Figure 2(d) shows the simulated transmission characteristics of the gap-adjustable directional coupler when the width of the waveguide changes to $400 \mathrm{~nm}$. It shows that optical switching occurs with $210 \mathrm{~nm}$ of gap size. Large extinction ratio (17.9 dB for drop port and $47.5 \mathrm{~dB}$ for through port) can still be achieved by changing the gap size between 550 and $210 \mathrm{~nm}$. Figure 2(e) shows optimal gap sizes for maximum power transfer to drop port for various widths of the waveguide. When the width changes from 400 to $500 \mathrm{~nm}$, one can still achieve optimal coupling with the gap sizes in the range from 210 to $90 \mathrm{~nm}$. Therefore, the dimensional accuracy for the waveguide can be relaxed during the fabrication process.

\section{Fabrication}

The switch can be fabricated using any standard "passive only" silicon photonics process. Figure 3 illustrates the fabrication flow we used. The fabrication starts with 200-mm SOI wafer 


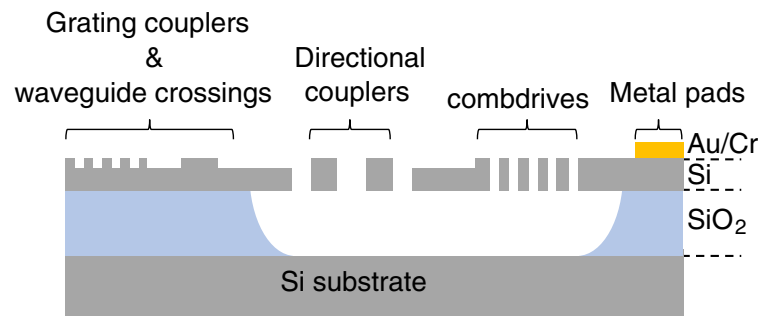

(a)

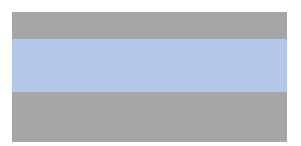

1. Start with SOI wafer

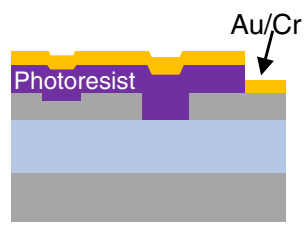

4. Lithography \& metal deposition

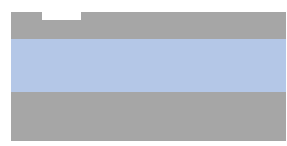

2. Lithography \& $70 \mathrm{~nm}$ deep Si etching

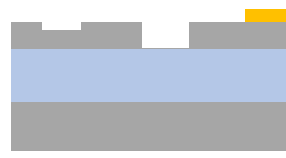

5. Metal lift-off

(b)

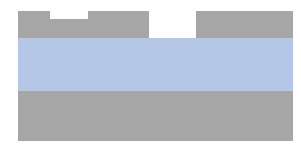

3. Lithography \& $220 \mathrm{~nm}$ deep Si etching

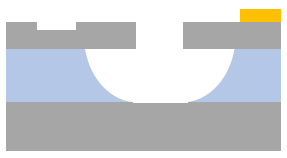

6. HF vapor etching

Fig. 3 (a) Cross section of the switch and (b) fabrication flow of the switch. (1) The fabrication starts with an SOI wafer. (2) First lithography and partial etching of the silicon layer with 70-nm depth. (3) Second lithography and full etching of silicon layer with 220-nm depth.

(4) Third lithography and metal (Au/Cr) deposition. (5) Metal pads patterned by lift-off process.

(6) Vapor HF etching to release the moving parts.

with 220-nm-thick silicon device layer and 3- $\mu$ m-thick buried-oxide (BOX) layer. The waveguides, grating couplers, directional couplers, and the comb-drive actuators are patterned by two lithographical steps: a partial and a full silicon etch processes.

A third lithography is used to define metal pads. As a last step, a hydrofluoric (HF) acid vapor is introduced to etch the BOX layer under the waveguide and the actuators for MEMS actuation. Figure 4(a) shows the scanning electron microscopy (SEM) image of the switch array fabricated in the foundry. Figure 4(b) shows the image of the full chip. The $32 \times 32$ switch matrix occupies an area of $5.9 \mathrm{~mm} \times 5.9 \mathrm{~mm}$. The grating couplers and routing waveguides occupy an additional area of $0.7 \mathrm{~mm} \times 8.4 \mathrm{~mm}$. Figures 4(c) and 4(d) show the optical and SEM images of the switch unit cell, respectively. Figure 4(e) shows the SEM image of comb fingers and spring. Figure 5(f) shows the SEM images of the directional coupler. There are 44 pairs of comb fingers in the comb-drive actuator. The width of the comb fingers is $300 \mathrm{~nm}$ and the gap between the comb fingers is $400 \mathrm{~nm}$. The suspended comb is supported by four folded springs. The width and the length of the springs are $300 \mathrm{~nm}$ and $30 \mu \mathrm{m}$, respectively. The thickness of the comb fingers and the springs are the same as the silicon device layer of the SOI wafer, which is $220 \mathrm{~nm}$. The initial gap of the directional coupler is $550 \mathrm{~nm}$. With this design, the switching voltage is calculated as $13.8 \mathrm{~V}$, and the resonance frequency of the actuator including all moving part is calculated as $58.4 \mathrm{kHz} .{ }^{24}$ The detailed calculation for the switching voltage and resonance frequency can be found in Ref. 25.

\section{Measurement}

Figure 5(a) shows the measured transmission characteristics of the switch unit cell with various applied voltages at $1550 \mathrm{~nm}$ wavelength. As the switch is designed for TE mode, all the measurement is done with the TE polarization. At $0 \mathrm{~V}$, optical transmission to the drop port is negligible (cell at off-state) and all the optical power goes to the through port. At $9.9 \mathrm{~V}$, the optical 


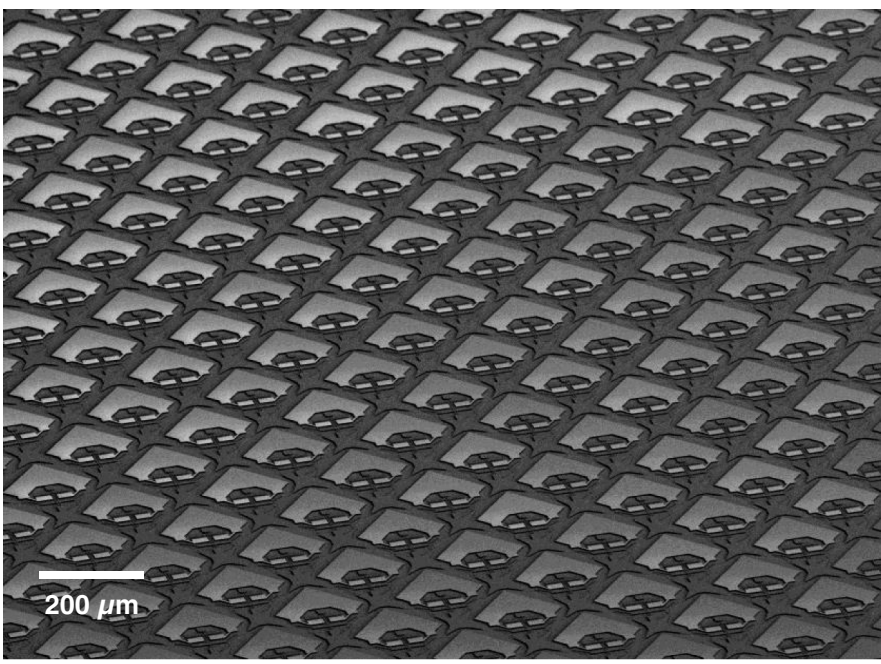

(a)

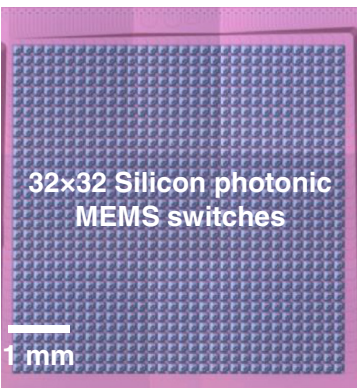

(b)

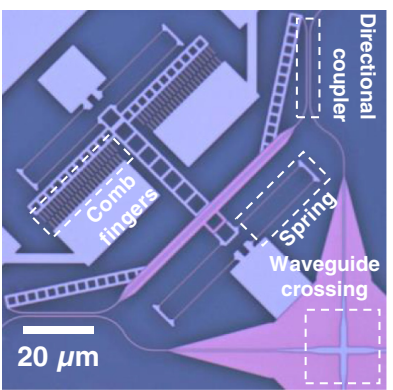

(c)

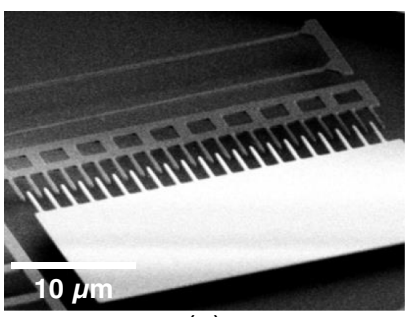

(e)

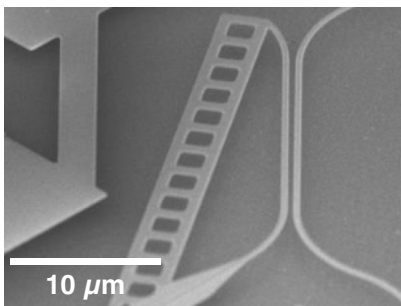

(f)

Fig. 4 Fabricated silicon photonic MEMS switches. (a) Perspective SEM image of the fabricated silicon photonic switch array. (b) Optical microscope image of the fabricated device. (c) Optical microscope image of the switch unit cell. In each unit cell, there are 2 pairs of the directional couplers, 4 folded springs, 44 pairs of comb fingers, and 1 waveguide crossing. (d) SEM image of the switch unit cell. (e) SEM image of the comb drive and spring. (f) SEM image of the directional coupler.

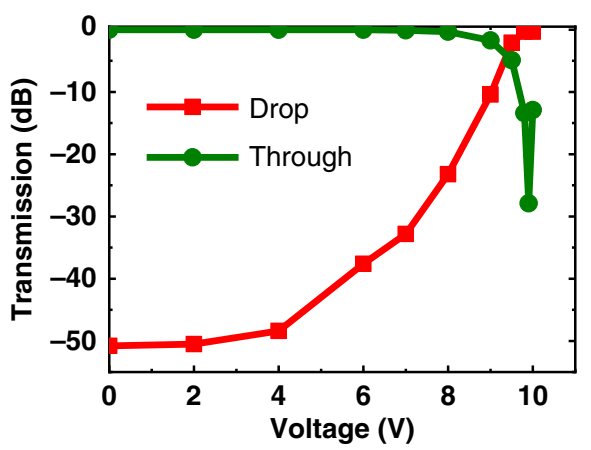

(a)

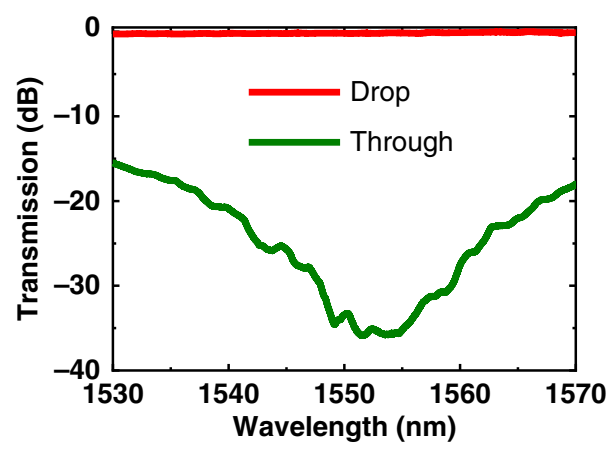

(b)

Fig. 5 (a) Measured transmission response of the switch unit cell with various applied voltages. (b) Measured spectral response of the switch unit cell when the unit cell is at on-state.

transmission to the drop port reaches maximum (cell at on-state), whereas the optical power transmitted to the through port is minimum. The on-off extinction ratios are $50.8 \mathrm{~dB}$ for the drop port and $27.9 \mathrm{~dB}$ for the through port. Figure 5(b) shows the spectral response of the switch unit cell when the unit cell is at on-state. The switching is optimized at near $1550 \mathrm{~nm}$ wavelength. The 20-dB-bandwidth of the through port is measured to be $28.7 \mathrm{~nm}$. 


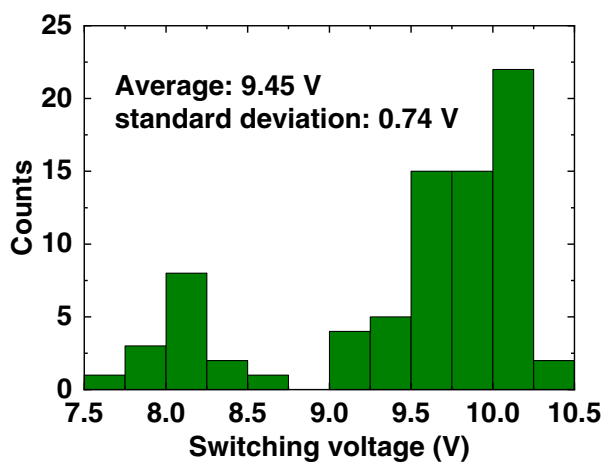

(a)

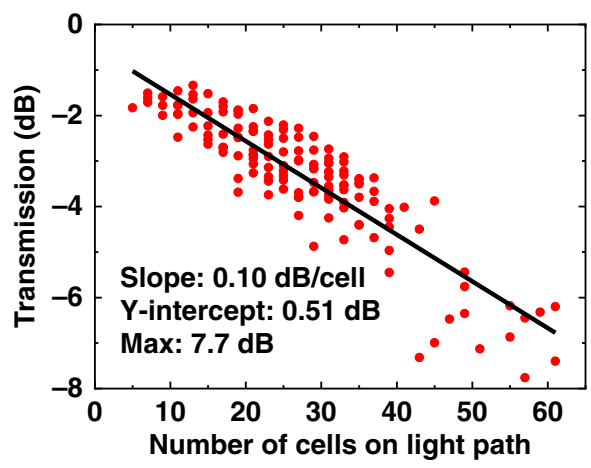

(b)

Fig. 6 (a) Distributions of the on-state voltage of the switch unit cells. (b) Measured pathdependent insertion of the switch.

Figure 6(a) shows the distributions of the on-state voltage of the switch unit cells. The average and the standard deviation of the switching voltages are 9.45 and $0.74 \mathrm{~V}$, respectively. The response time of the switch was measured as $50 \mu \mathrm{s}$, which is longer than the value inferred from the resonant frequency of the actuator (i.e., $58.4 \mathrm{kHz}$ ). This is because the high resistivity of the wafer substrate caused a large RC delay in the actuator. However, as demonstrated in our previous work, ${ }^{24}$ using a wafer with a low-resistive substrate can reduce the switching time below $10 \mu$ s with the same actuator design. We measured various optical paths in the $32 \times 32$ switch to find the path dependence insertion loss of the switch [Fig. 6(b)]. The loss per unit cell is $0.10 \mathrm{~dB}$, extracted from the slope of the linear fit line of the data. The $y$ intercept $(=0.51 \mathrm{~dB})$ includes the optical losses of the two directional couplers at on-state and the optical losses of the routing waveguides to/from the grating couplers. Therefore, the fixed switching loss is $<0.51 \mathrm{~dB}$. The maximum on-chip insertion loss for the $32 \times 32$ switch is only $7.7 \mathrm{~dB}$. There are optical test structures for measuring the propagation loss of the waveguides with cut-back method. The propagation loss of the 450-nm wide fully etched waveguide (same dimension as the waveguide used in the directional couplers) and the $450-\mathrm{nm}$ wide shallow etched waveguide is measured as 1.7 and $0.8 \mathrm{~dB} / \mathrm{cm}$, respectively. Insertion loss of the grating coupler is measured as $3.8 \mathrm{~dB}$ at $1550 \mathrm{~nm}$ wavelength.

\section{Conclusion}

In summary, we have demonstrated $32 \times 32$ silicon photonic MEMS switch on a single-layer silicon-on-insulator substrate with gap-adjustable directional couplers. The switch chip is successfully fabricated at a commercial CMOS foundry on 200-mm wafers. The switch array area is $5.9 \mathrm{~mm} \times 5.9 \mathrm{~mm}$. The use of a foundry-based silicon photonic process allows the switch to be intimately integrated with other silicon photonic integrated circuits. The continuously tunable gap allows us to compensate for the variation of the waveguide width in the directional couplers, though this does require a customized bias for each switch cell to achieve the best extinction ratio. One way to control the bias precisely is to use the through port signal for feedback control. Alternatively, the need for precise bias control can be alleviated by replacing directional couplers with adiabatic couplers, as in our other reported switches. ${ }^{15,21}$ The operating voltages of the switch cell are quite uniform (standard deviation $=0.74 \mathrm{~V}$ ), and the maximum on-chip insertion loss of the switch is only $7.7 \mathrm{~dB}$. We believe that this work shows a promising path for mass production of large-scale silicon photonic switches at commercial foundries.

\section{Acknowledgments}

This work was supported by DARPA PIPES (No. HR0011-19-2-0015), ARPA-E ENLITENED (No. DE-AR0000849); National Science Foundation (NSF), Center of Integrated Access Networks (CIAN), Engineering Research Center (No. EEC-0812072), and Partnership for 
Innovation-Technology Transition (PFI-TT) (No. 1827633); Google Faculty Research Award; and UC Berkeley Bakar Fellow program. The authors declare that there are no conflicts of interest.

\section{References}

1. D. Amodei and D. Hernandez, "AI and compute. Blog post, OpenAI," 2018, https://openai .com/blog/ai-and-compute/.

2. Q. Cheng et al., "Silicon photonic switch topologies and routing strategies for disaggregated data centers," IEEE J. Sel. Top. Quantum Electron. 26(2), 8302010 (2020).

3. W. M. Mellette et al., "RotorNet: a scalable, low-complexity, optical datacenter network," in Proc. ACM Spec. Interest Group on Data Commun., New York, pp. 267-280 (2017).

4. B. G. Lee et al., "Silicon photonic switch fabrics in computer communications systems," J. Lightwave Technol. 33(4), 768-777 (2015).

5. G. Porter et al., "Integrating microsecond circuit switching into the data center," in Proc. ACM SIGCOMM 2013 Conf., Vol. 43(4), pp. 447-458 (2013).

6. J. Bowers et al., "Advantages and control of hybrid packet optical-circuit-switched data center networks," in Adv. Photonics for Commun., Optical Society of America, p. PM2C.4 (2014).

7. K. Suzuki et al., "Broadband silicon photonics $8 \times 8$ switch based on double-Mach-Zehnder element switches," Opt. Express 25(7), 7538 (2017).

8. K. Suzuki et al., "Low-insertion-loss and power-efficient $32 \times 32$ silicon photonics switch with extremely high- $\Delta$ silica PLC connector," J. Lightwave Technol. 37, 116-122 (2018).

9. R. Konoike et al., "Ultra-compact silicon photonics switch with high-density thermo-optic heaters," Opt. Express 27(7), 10332 (2019).

10. N. Dupuis et al., "Nanosecond-scale Mach-Zehnder-based CMOS photonic switch fabrics," J. Lightwave Technol. 35(4), 615-623 (2017).

11. J. E. Proesel et al., "A monolithically integrated silicon photonics $8 \times 8$ switch in $90 \mathrm{~nm}$ SOI CMOS," in IEEE Symp. VLSI Circuits, Honolulu, Hawaii, IEEE (2020).

12. N. Dupuis et al., "A $4 \times 4$ electrooptic silicon photonic switch fabric with net neutral insertion loss," J. Lightwave Technol. 38(2), 178-184 (2020).

13. Q. Li et al., "Ultra-power-efficient $2 \times 2 \mathrm{Si}$ Mach-Zehnder interferometer optical switch based on III-V/Si hybrid MOS phase shifter," Opt. Express 26(26), 35003 (2018).

14. N. Dupuis et al., "An $8 \times 8$ silicon photonic switch module with nanosecond-scale reconfigurability," in Opt. Fiber Commun. Conf., p. Th4A.6 (2020).

15. T. J. Seok et al., "Wafer-scale silicon photonic switches beyond die size limit," Optica 6(4), 490 (2019).

16. N. Quack et al., "MEMS-enabled silicon photonic integrated devices and circuits," IEEE J. Quantum Electron. 56, 8400210 (2019).

17. S. Han et al., "Large-scale silicon photonic switches with movable directional couplers," Optica 2(4), 370-375 (2015).

18. T. Nagai and K. Hane, "Silicon photonic microelectromechanical switch using lateral adiabatic waveguide couplers," Opt. Express 26(26), 33906 (2018).

19. S. Han et al., "Multicast silicon photonic MEMS switches with gap-adjustable directional couplers," Opt. Express 27(13), 17561 (2019).

20. W. Bogaerts et al., "MORPHIC: programmable photonic circuits enabled by silicon photonic MEMS," Proc. SPIE 11285, 1128503 (2020).

21. T. J. Seok et al., "Large-scale broadband digital silicon photonic switches with vertical adiabatic couplers," Optica 3(1), 64-70 (2016).

22. H. Chu and K. Hane, "A wide-tuning silicon ring-resonator composed of coupled freestanding waveguides," IEEE Photonics Technol. Lett. 26(14), 1411-1413 (2014).

23. Y. Akihama and K. Hane, "Single and multiple optical switches that use freestanding silicon nanowire waveguide couplers," Light Sci. Appl. 1, e16 (2012).

24. S. D. Senturia, Microsystem Design, 1st ed., Springer US (2001). 
25. S. Han et al., "Multicast silicon photonic MEMS switches with gap-adjustable directional couplers," Opt. Express 27(13), 17561 (2019).

Sangyoon Han received his $\mathrm{PhD}$ in electrical engineering and computer sciences from the University of California, Berkeley, in 2016. From 2016 to 2020, he was at Korea Advanced Institute of Science and Technology (KAIST), Daejeon, Republic of Korea. He has been an assistant professor at Daegu Gyeongbuk Institute of Science and Technology, Daegu, Republic of Korea, since 2020. His research interests include optoelectronics, silicon photonics, and MEMS. He was the recipient of the Bronze Medal of Collegiate Inventors Competition.

Jeremy Beguelin received his MSc degree in physics and his $\mathrm{PhD}$ in photonics from the Ecole Polytechnique Fédérale de Lausanne (EPFL) in 2016 and 2020, respectively. For his master thesis, he worked on optical MEMS as a visiting scholar at UC Berkeley in the integrated photonics laboratory. He is currently an R\&D engineer at SUSS MicroOptics working on refractive microlenses.

Lane Ochikubo received his BS degree in electrical and computer engineering from the University of California, Irvine in 1991. He is a principal process engineer at TSI Semiconductors. His semiconductor specialty is photolithography.

John Jacobs received his BS degree in chemical engineering from the University of California, Berkeley, in 1992. He performed both development and manufacturing work for NEC Electronics, Inc. from 1992 until 2011 and has been performing development work for TSI Semiconductors since 2011. He is a senior engineering manager at TSI Semiconductors. He is an author on several journal papers and an author on several USA patents. His semiconductor specialties include plasma and wet etching.

Tae Joon Seok received his $\mathrm{PhD}$ in electrical engineering and computer sciences from the University of California, Berkeley, in 2012. From 2013 to 2016, he was at the UC Berkeley. He has been a professor at Gwangju Institute of Science and Technology, Gwangju, Republic of Korea, since 2017. His research interests include integrated photonics, silicon photonics, and optoelectronics. He was the recipient of the Tingye Li Innovation Prize and the Bronze Medal of Collegiate Inventors Competition.

Kyoungsik $\mathrm{Yu}$ received his $\mathrm{MS}$ and $\mathrm{PhD}$ degrees in electrical engineering from Stanford University in 2001 and 2004, respectively. From 2004 to 2007, he was at Korea Electrical Engineering and Science Research Institute, Seoul, Republic of Korea. From 2007 to 2009, he was a postdoc at UC Berkeley. He joined the School of Electrical Engineering at KAIST, Daejeon, Republic of Korea, in 2010. His research interests are micro- and nano-photonics, sensing, and imaging.

Niels Quack received his MSc degree from EPFL, Lausanne, Switzerland, in 2005 and his DrSc degree from ETH Zürich, Zürich, Switzerland, in 2010. He is an SNSF assistant professor at the EPFL. He has authored and co-authored more than 50 papers in leading technical journals and conferences. His research focuses on photonic micro- and nanosystems. He is a senior member of IEEE, a member of The Optical Society (OSA), and a life member of SPIE.

Chang-Kyu Kim received his BS, MS, and PhD degrees in physics from KAIST, Daejeon, Republic of Korea. He is a professor at Korea Polytechnic University, Siheung, Republic of Korea. Before joining Korea Polytechnic University, he was at the Electronics and Telecommunications Research Institute, Gwangju, Republic of Korea. His research interests include MEMS and photonics.

Richard S. Muller received his MS degree in electrical engineering and his $\mathrm{PhD}$ in electrical engineering and physics from the California Institute of Technology in 1957 and 1962, respectively. He is currently an emeritus electrical engineering and computer science professor at UC Berkeley. He received several awards, including Cledo Brunetti Award and James Clerk Maxwell Award. He was elected to membership in the National Academy of Engineering in 1994. 
Han et al.: $32 \times 32$ silicon photonic MEMS switch with gap-adjustable directional couplers fabricated...

Ming C. Wu received his PhD from UC Berkeley in 1988. He is a Nortel Distinguished Professor of electrical engineering and computer sciences at the University of California, Berkeley and a co-director of Berkeley Sensor and Actuator Center. Before joining Berkeley faculty, he was at AT\&T Bell Labs and UCLA. His research focuses on MEMS, optofluidics, optoelectronics, and nanophotonics. He is IEEE and OSA fellow. He received several awards including Robert Bosch MEMS award from IEEE. 